\title{
La respuesta de la economía urbana al Tratado de Libre Comercio de América del Norte: planificar para la competitividad'
}

Peter Karl Kresl

Universidad de Bucknell

\section{Resumen}

continúa

1 Traducción realizada por el Dr. Daniel Hiernaux Nicolas, profesor de la Universidad Autónoma Metropolitana, Unidad Xochimilco. 
mientras que las actividades económicas son más móviles y pueden ser relocalizadas con más facilidad que en otros tiempos.

Los factores de localización tradicionales (recursos, factores de la producción, proximidad al transporte y la aglomeración), han dejado de ser decisivos. Ahora las políticas son cada vez más decisivas como factores locacionales.

En este contexto, el artículo analiza tres hipótesis. La primera plantea que, como efecto de la liberalización y el cambio tecnológico, las ciudades han surgido como actores económicos de primer orden, al tiempo que los Estados-nación han retrocedido. La segunda hipótesis es que las ciudades pueden diseñar respuestas estratégicas a los retos y las oportunidades. Finalmente, se plantea que las ciudades pueden poner en práctica políticas y realizar inversiones que les permitan desarrollar su potencial.

De esta forma, las opciones de las ciudades para planificar su desarrollo, estratégicamente en este contexto, son de dos tipos: la reestructuración cualitativa de sus actividades o la expansión cuantitativa con la misma estructura productiva básica.

En ambos casos, la ciudad requiere cambios institucionales y de inversiones. Ambos caminos son estrategias dentro de la competitividad. Sin embargo, también es posible un tercer camino: la cooperación entre ciudades.

Las ciudades o las economías urbanas, nunca recibieron mucha atención por parte de los especialistas en economía internacional; desde Adam Smith, nuestra atención ha sido canalizada hacia "la nación”. Después de todo, es el “bienestar de las naciones" lo que trató Smith; por su parte, Friedrich List escribió acerca del "sistema nacional de la economía política" y Michael Porter ha analizado recientemente la "ventaja competitiva de las naciones". A pesar de ello, durante la década actual, el lugar de las ciudades en la economía global ha empezado a emerger como un tópico relevante para el trabajo intelectual. Para tomar sólo dos ejemplos, el libro de Paul Krugman Geografía y Comercio plantea una justificación teórica en relación al interés por los niveles territoriales sub-nacionales y la
Organización para la Cooperación para el Desarrollo Económico (OCDE) ha publicado recientemente los textos presentados, en noviembre de 1995, en la conferencia titulada "Las ciudades en la nueva economía global".

Este interés reciente por la internacionalización de las economías urbanas es una respuesta a los cambios en el contexto económico en el cual las ciudades se ubican y en el cual deben funcionar. Adam Smith alertó a sus lectores acerca de la declinación del mercantilismo y el surgimiento de una economía de mercado liberal; el argumento central de este ensayo se basa en el planteamiento de que existen cambios fundamentales de poder y de responsabilidades mutuas con respecto al pensamiento económico estratégico, entre el Estado-nación y la ciudad. Los cambios en la economía mundial, que se encuentran atrás de estos cambios en la distribución del poder, serán desarrollados en la primera sección sustantiva de este ensayo. En la segunda, el trabajo se orientará hacia las ciudades en sí y los retos estructurales que deben afrontar. Las posibilidades de una respuesta creativa, por parte de los líderes de una ciudad, se analizarán posteriormente; el texto concluirá con algunas sugerencias para las próximas décadas en relación a la naturaleza de la situación económica emergente para las economías urbanas en la economía global.

\section{El contexto económico global}

Mientras la mayor parte de las ciudades eran esencialmente autónomas hasta el siglo XVII, el periodo de construcción de la nación, concomitante con el mercantilismo, estuvo marcado por la subordinación de la vida económica de las ciudades a un nivel más elevado de gobierno. Al pensar en términos de economía internacional, se fue haciendo cada vez menos referencia a las ciudades.

Los gobiernos centrales impusieron un abanico de barreras comerciales, establecieron procesos centralizados de planificación económica y de coordinación y, en forma creciente durante el siglo $\mathrm{XX}$, asumieron la responsabilidad del manejo macroeconómico; por su parte, el nivel de decisión microeconómico fue dejado a la empresa. Las ciudades no fueron totalmente pasivas, pero sus iniciativas de políticas quedaron orientadas al mejoramiento de los problemas urbanos como la congestión del tránsito, la contaminación ambiental,

${ }^{2}$ Krugman, Paul (1991), Geography and trade, Cambridge, MrT Press, 1991; y OCDE (1995), Cities and the New Global Economy, Camberra OCDE y Commonwealth of Australia. 
el crimen y la pobreza, o hacia el mejoramiento de su atractividad, mediante centros de actividades bajo la responsabilidad de los centros urbanos, como lo referente a la vida cultural de la nación. Sin embargo, la planificación estratégica para el desarrollo económico y el mejoramiento de la competitividad, quedaron fuera de su alcance.

\section{La liberalización comercial}

Sin embargo, a partir de los años treinta, una tendencia en sentido contrario empezó a surgir. Se dijo que el proteccionismo había ensanchado y prolongado la Gran Depresión y, mientras que algunos esfuerzos de liberalización fueron tomados tempranamente, en 1934, no fue sino hasta después de la Segunda Guerra Mundial, cuando serias iniciativas multinacionales fueron introducidas para desmantelar la estructura del proteccionismo. Por medio del Acuerdo General de Tarifas y Preferencias (GATT), las naciones más grandes del mundo llegaron a entendimientos para reducir los aranceles, restringir severamente otras medidas proteccionistas, definir lo que debe ser un comportamiento comercial aceptable y establecer un mecanismo imparcial de resolución de disputas.

Este ímpetu hacia una liberalización del comercio, fue acelerado con la sustitución del GATT por la Organización Mundial del Comercio en 1994. La importancia de esta tendencia hacia la liberalización de los flujos de bienes y servicios, a lo largo de la economía global, surge como consecuencia de que a lo largo de este proceso, las naciones fueron imponiendo restricciones a su capacidad de intervención en favor de sus propias estructuras domésticas, que no eran capaces de enfrentar la competencia externa. Mientras que los países aún tienen una enorme influencia gracias al manejo de la tasa de interés, la tasa de cambio, la competencia y las políticas industriales; en forma lateral éstas se están volviendo menos significativas en la medida que se agudiza la competencia económica sobre una base global.

El ímpetu global hacia la liberalización del comercio ha sido respaldado por varias iniciativas a nivel regional. En Europa, por ejemplo, el "proceso de 1992" y el Tratado de Maastricht, han profundizado la integración económica y política, al grado de empezar a tomar en consideración la integración monetaria y la expansión de la membresía a 15 naciones para este bloque económico, con otros países inclusive esperando su turno. Canadá, los Estados Unidos y México, en contraste, han limitado su esquema de liberalización económica a un área de libre-comercio, pero han instituido un mecanismo imparcial de resolución de disputas y negociado acuerdos complementarios sobre las condiciones laborales y de medio ambiente. Así, cada agrupación regional ha negociado un pacto de integración de conformidad con sus condiciones específicas, sin embargo, cada una ha impuesto restricciones a la capacidad de los gobiernos nacionales para intervenir en sus propias actividades económicas.

Al mismo tiempo, en la medida en que las naciones ven disminuida, en cierta manera, su capacidad de intervención, las ciudades emergen como actores económicos de importancia creciente, en espacios económicos que son de alcance continental o global. La ciudad que es capaz de usar esta nueva libertad de acción para sus propósitos, tendrá una mejor posibilidad de realizar las aspiraciones de sus ciudadanos y se volverá la localización más deseada para la actividad económica. Esta posibilidad de acción, asignada a los líderes de la ciudad, será explorada extensamente en apartados posteriores.

\section{El desarrollo tecnológico}

Las décadas posteriores a la Segunda Guerra Mundial, fueron también testigo del desarrollo exponencial de la tecnología que forzó a una reevaluación de las consecuencias de los procesos de producción, transporte y comunicación, en relación a la localización de la actividad económica. Grandes "distritos industriales", como los centros de producción de automóviles o de acero, han cedido el paso a minifundiciones, la subcontratación y la producción globalizada. ${ }^{3} \mathrm{La}$ reestructuración consecuente ha generado nuevas oportunidades de empleo y producción en las nuevas economías regionales favorecidas por estos procesos, aunque lo anterior ha sido contrarrestado por los impactos negativos de la misma magnitud en centros tradicionales de producción. Las economías de aglomeración, se han vuelto menos decisivas, en muchos casos, con relación a la proximidad de los mercados. Los avances en materia de transporte y de tecnologías de comunicación, han significado que el ordenamiento espacial de las actividades deba ser repensado y sometido más directamente a otras consideraciones, tales como las facilidades para ciertos modos de vida y ciertas preferencias residenciales. La empresa en sí, puede ser desen-

${ }^{3}$ Para un punto de vista escéptico sobre los distritos industriales, véase. Harrison, Bennett, "Industrial Districts: Old Wines in new Bottles?", Regional Studies, Vol. 226, No 5, pp. 469 . 483. 
samblada de tal suerte, que cada actividad se localice óptimamente en relación a sus necesidades específicas, en vez de estar organizada en grupos (cluster) para ejercer el control y la coordinación. Estas últimas funciones ahora, pueden ser efectuadas por medios electrónicos desde localizaciones distantes.

Lo anterior es de gran importancia para las ciudades por diversas razones: en primer lugar, las actividades económicas se han vuelto bastante móviles y pueden ser relocalizadas en plazos muy breves. En segundo lugar, las ciudades deben hacer esfuerzos para retener las actividades económicas que consideran deseables para su entorno. En tercer lugar, las ciudades tienen ahora, la posibilidad de crear una ventaja comparativa en vez de estar limitadas a la disponibilidad de los factores tradicionales tales como recursos o los factores de producción, la proximidad de los medios de transporte o el factor de aglomeración. Las políticas que son adoptadas por las ciudades en forma individual, tienen cada vez más importancia; sus expectativas económicas a futuro están influidas en forma creciente por las decisiones tomadas por los líderes locales, tanto en el sector privado como público.

\section{El reto para las ciudades}

La liberalización del comercio y el cambio tecnológico, en los años posteriores a la Segunda Guerra Mundial, se constituyeron en las fuerzas primarias del cambio. Sus impactos sobre la situación económica de las ciudades, han sido numerosos, aunque en este trabajo queremos centrarnos sobre dos de ellos, los cuales, desde una perspectiva más abierta que la neoclásica, son los más interesantes $\mathrm{y}$, posiblemente, los más significativos.

\section{Los nuevos espacios económicos}

Ambas fuerzas de cambio (la liberalización del comercio y el cambio tecnológico) han hecho obsoletas las restricciones existentes sobre el espacio económico. Numerosas economías urbanas se han beneficiado con las políticas del gobierno central, quien es el que ha protegido sus mercados tradicionales de la competencia extranjera. En la medida en que estas barreras han sido reducidas progresivamente a lo largo de las rondas de negociaciones del GATT y en numerosas partes del mundo mediante los acuerdos regionales como el TLCAN y la Unión Europea de 1992, estos mercados, relativamente seguros, han sido abiertos y el espacio económico, en el cual la ciudad encuentra sus nichos competitivos, se han ensanchando hasta cubrir un continente o se han vuelto auténticamente intercontinentales. Unos cuantos ejemplos servirán para explicar el tipo de impactos resultantes.

Cuando Suecia votó su integración a la Unión Europea, la decisión de establecer el puente öresund entre Copenhague y Malmö, se perfiló como una decisión sensata. La membresía de ambos países en el Consejo Nórdico y las relaciones entre la Unión Europea y EFTA, dan sentido a la relación entre las mayores ciudades de Dinamarca y del Sur de Suecia, pero con las reforzadas posibilidades de cooperación política y económica entre miembros de la Unión Europea, esta integración transfronteriza tendrá avances sustanciales. El resultado será un nuevo patrón de trabajo, de residencia, de actividades comerciales y de recreación.

En América del Norte, Seattle-Vancouver, Toronto-Buffalo y San Diego-Tijuana son ejemplos de este proceso, como en Europa lo son Maastricht-Liège-Aachen y el "Basel Dreiecke". Cada una de estas ciudades ha pasado por la experiencia de una limitación de su alcance económico, debido a la imposición de una frontera nacional arbitraria. La reducción del significado de la frontera ha permitido a la ciudad expandir el mercado de sus productos al área contigua y también le ha permitido ubicarse en mercados de trabajo y de capital reorganizados, así como reestructurar sus sistemas de transporte.

A una mayor escala, Londres, Tokio y Nueva York han sido descritas como "ciudades globales" ${ }^{4}$ y decenas de ciudades de menor rango, han encontrado espacios continentales o globales para su actividad económica específica, de los cuales pueden sacar provecho. Amsterdam, Hamburgo, Seattle, Miami, Singapur y Osaka vieron estimuladas sus economías por las consecuencias de la globalización.

\section{Nuevas relaciones de cooperación y competencia}

En la medida en que el espacio económico de cada ciudad se ha expandido, éstas han entrado en contacto entre sí en grados que no se conocían en el pasado. La primera consecuencia del incremento del contacto entre ciudades, es el reconocimiento de la relación de com-

4 Éste es el nombre que les asignó Sassen en su obra: Sassen, Saskia (1991), The Global City. New York, London, Tokio. Princeton, New Jersey, Princeton University Press. 
petencia que existe forzosamente entre los centros de producción y distribución, en cualquier economía de mercado. Las ciudades compiten por la localización de las industrias, por lograr eventos como los Juegos Olímpicos, por facilidades de transporte, por el gasto turístico, por las sedes sociales de empresas e instituciones y últimamente, por una posición en la jerarquía internacional de ciudades. La segunda consecuencia, sin embargo, es el descubrimiento de oportunidades considerables para una cooperación de mutuo beneficio, a veces en proyectos específicos, como trenes de alta velocidad, pero más frecuentemente en el campo más general del intercambio de experiencias, y de la discusión de las soluciones a problemas comunes, como la desindustrialización o el impulso a la proyección internacional de las pequeñas y medianas empresas. ${ }^{5}$

La frontera entre los Estados Unidos y Canadá demuestra una clara evidencia de ambos modelos de interacción. Mientras que la mayor parte de los contactos transfronterizos han sido marcados por la competencia e inclusive por el conflicto en temas como el aprovechamiento de los recursos forestales, las pesquerías y la producción agrícola; el segmento de la frontera Grandes Lagos-Valle del San Lorenzo ha sido dominado por la cooperación, las articulaciones de beneficio mutuo y la especialización intraindustrial. Las áreas de conflicto se caracterizan por dos economías que esencialmente producen, para el mismo mercado, bienes no diferenciados, pero incluso así, las ciudades de Seattle y Vancouver por ejemplo, han creado un alto grado de cooperación en materia económica y cultural. En Europa, el movimiento de las Eurocities y Eurométropoles ha establecido asociaciones formales dedicadas a la intervención conjunta interurbana y a la solución de los problemas.

Para una cooperación efectiva y para la realización de los beneficios potenciales de la competencia entre ciudades, es necesario que las ciudades se percaten, en forma individual, de sus funciones específicas en la economía global y adopten políticas que les permitan desarrollar a fondo su potencial económico para el beneficio del empleo e ingreso de sus habitantes y de sus unidades de producción. Ahora, nos dedicaremos a las acciones éspecíficas que pueden tomar las ciudades.

${ }^{5}$ Para un conjunto de artículos que repasen este fenómeno, véase "Urban networking in Europe", Fkistics, Vol.58, N0350/351, septiembre/octubre y noviembre/diciembre de 1991 y vol.59, No352/353, enero/febrero y marzo/abril de 1992.

\section{La respuesta de las ciudades}

Los retos impuestos a las ciudades por la liberalización del comercio y el cambio tecnológico, no plantean la necesidad de una respuesta por parte de cada ciudad, aunque hoy resulta extremadamente arriesgado para una ciudad dejar que su economía se maneje pasivamente, frente a las implicaciones que puedan dictarle cada función nueva, cada ventaja comparativa o las fuerzas corporativas. La lógica de este argumento es particularmente evidente cuando se recuerda, que no es imaginable que los otros actores, públicos o privados, hagan otra cosa distinta que lo que están haciendo activamente para su propio interés. Las empresas toman sus decisiones de producción sobre la base de la información disponible y mientras buena parte de esta información es objetiva (la fiscalidad impuesta al capital y los niveles de salario, por ejemplo), los diversos niveles de gobierno influyen las variables claves, que pueden ser decisivas en la toma de las decisiones. Esto se evidenció cuando Atlanta fue seleccionada, en vez de Toronto, para las Olimpíadas de Verano, así como en el caso de las plantas de producción de automóviles alemanes que fueron localizadas en el Sur-Este de los Estados Unidos, en vez serlo en el "Norte industrial". Se ve entonces que surge un costo sustancial para cualquier ciudad que se mantiene pasiva o que no puede plantear un liderazgo efectivo para su propio desarrollo económico. La respuesta de una ciudad a la globalización de su entorno económico, debe ser definida en relación a lo que suele llamarse la "planeación estratégica" ola "competitividad de las economías urbanas". Para esta discusión, basaremos nuestros comentarios en los resultados de dos de nuestras investigaciones recientes.

\section{La planificación estratégica}

La noción esencial que está detrás de la planificación estratégica para las economías urbanas, es que una ciudad dispone de más de una orientación para su desarrollo futuro y que el liderazgo de la ciudad puede poner en práctica políticas e iniciativas que le permitirán seguir la orientación que más beneficie a las empresas y a los habitantes de esta ciudad. La planificación estratégica implica:

- Una evaluación objetiva de la situación en la cual se desempeña la ciudad, 
- Una estimación realista de las fuerzas y las debilidades de la ciudad,

- La determinación de la mejor estrategia de desarrollo,

- La especificación de lo que debe hacerse para poner en práctica esta estrategia y,

- Un claro entendimiento tanto de los beneficios que se pueden alcanzar como de los costos para lograrlos.

Este trabajo no es el lugar adecuado para desarrollar cada paso del proceso de planificación, ni resultaría útil para nuestro objetivo actual, sin embargo podemos especificar algunas de las condiciones básicas de su desempeño. ${ }^{6}$ Después será posible examinar un marco conceptual para las decisiones que deben tomar los líderes de las ciudades y finalmente, presentar la experiencia de algunas ciudades de Europa y América del Norte.

La teoría económica nos enseña que la actividad económica de cualquier región o nación, está determinada por factores como su localización, la disponibilidad de recursos, la calidad de la fuerza de trabajo, la proximidad a los mercados, la actividad de otras regiones o naciones, la eficacia en la toma de las decisiones y el liderazgo. Con escasas excepciones, estos factores no implican que la ciudad tenga frente a ella una sola opción para su futuro, sino que dispone de una gama de ellas, y el reto para la ciudad es el de elegir la opción que le brindará los mayores beneficios netos. El abanico de opciones que se abre para una ciudad es demasiado amplio como para ser enlistado aquí, pero podemos entender su extensión al examinar la figura 1. Que el objetivo último sea la vitalidad económica es innegable, pero para lograr estos objetivos, algunas ciudades elegirán continuar con la estructura básica de su actividad económica, expandiendo su nivel de actividad; mientras que otras elegirán la reestructuración cualitativa de su actividad y luchar esencialmente para ubicarse en nuevas opciones.

Ya sea que la ciudad encuentre que la expansión cuantitativa - la reestructuración cualitativa de su actividad es su objetivo más apropiado, para cada opción existe una gama de "estrategias" disponi-

6 Ofrecemos mayor detalle a este respecto en Kresl, Peter Karl (1992), The Urban Economy and Regional Trade Liberalization, Praeger, Nueva York. bles, entre las cuales la ciudad debe elegir una o varias para integrar su plan estratégico. En la figura 1, se plantean cuatro estrategias para cada uno de los objetivos, y a pesar de que algunos se entiendan sin mayor explicación, elaboraremos algunas observaciones sobre cada una de ellas:

\section{- La promoción de las exportaciones}

Cuando una ciudad tiene un sector manufacturero bien desarrollado, cuya producción es aún competitiva, puede ser racional elegir una política de desarrollo posterior de esta actividad basada en un crecimiento sustentado en ventas hacia nuevos mercados.

\section{- La ciudad-puente}

Una ciudad-puente es aquella que se sitúa típicamente entre dos mercados y busca mejorar su utilidad como canal de circulación de bienes, servicios y personas entre ambos mercados. Puede ser posible para la ciudad-puente desarrollar facilidades para el transporte de bienes, para el ensamble y para la distribución; de tal suerte que desarrolle actividades que incrementen el valor agregado.

\section{- Centro regional}

Con la creación de nuevos espacios económicos, algunas ciudades encontrarán la posibilidad de volverse el centro de coordinación de una región para una economía que se ha constituido más como continental que nacional, en cuanto a su alcance. Estos nuevos espacios económicos pueden ser regiones transfronterizas, entre dos o más países, o pueden ser parte de áreas geográficas internas a un país, que se encuentran en un proceso de interacción incrementada con otras partes de la economía global. 
- Sedes sociales nacionales

A pesar de que las naciones han disminuido en relevancia, como actores económicos en la economía global en desarrollo, numerosas empresas continúan queriendo estructurar sus actividades sobre una base nacional. Lo anterior se debe a que, para muchas empresas, la regulación gubernamental, el apoyo recibido, la política de cambio, los incentivos a la localización y los costos de transporte, siguen ocupando un lugar privilegiado en sus decisiones. Entonces, las ciudades con una fuerza tradicional en ciertas ramas de la producción o distribución, tienen la posibilidad de volverse el sitio de localización de las sedes sociales de estas firmas.

\section{- Desarrollo del nicho}

Para que una economía manufacturera sobreviva a una competencia acrecentada, será necesario que restrinja la variedad de su línea de producción y que se especialice en "nichos" o segmentos estrechos de la industria. Si se logra lo anterior, la economía urbana será capaz de sobrevivir sin la necesidad de emprender una costosa y penosa transición hacia una nueva base económica.

\section{- Ciudad-punto de acceso}

Mientras que la ciudad-puente ofrece un conducto para los productos y servicios entre dos mercados y puede aspirar a algunas actividades de baja incorporación de valor agregado, las ciudades-punto de acceso sirven de base operativa para las empresas de cualquier parte del mundo que busquen el acceso a un mercado continental, como el norteamericano o el europeo. Esta función implica la localización de ejecutivos de alto nivel y de trabajadores profesionales especializados.

\section{- Centro de Investigación y Desarrollo}

Una consecuencia de la reestructuración es que las empresas están en posibilidad de fragmentar sus actividades y relocalizar cada componente en forma óptima. La investigación y el desarrollo son activi- dades deseables porque tienen efectos ambientales mínimos, implican la presencia de trabajadores de alta calificación e ingreso y sus actividades tienden a ser relativamente independientes de los altibajos de la economía, porque atraen a otras empresas e instituciones interesantes. Para tener éxito como Centro de Investigación y Desarrollo, una ciudad debe, típicamente, tener una base de crecimiento, como universidades u otras estructuras de investigación.

\section{- Sedes sociales internacionales}

Mientras que muchas ciudades actúan como centro internacional de empresas individuales que sostienen relaciones históricas con la comunidad local, sólo unas cuantas pueden ser el centro de actividad de las sedes internacionales de un gran número de empresas.

Algunos conjuntos de estrategias son más apropiados para objetivos distintos, sin embargo, difieren en forma más relevante en los "medios" que se usarán para su realización. Otra vez, la figura 1 permite aclarar este punto. Los medios enlistados, de I a VIII, representan conjuntos de iniciativas para las instituciones y gastos que deben realizarse para que la ciudad sea exitosa en lograr la estrategia o combinación de estrategias que considere más apropiada para su situación peculiar. Comparemos, por ejemplo, los medios requeridos para una ciudad-puente y una ciudad-punto de acceso. Mientras que ambas requieren de gastos en infraestructura de transporte, la necesidad de una ciudad-puente se sitúa en las autopistas y los puentes que facilitarán el movimiento a través de su área y que permitirán vincular las dos economías regionales o nacionales. La ciudad-punto de acceso requerirá de un aeropuerto con vuelos internacionales intercontinentales, así como de buenas conexiones directas hacia las demás partes del espacio económico continental. La ciudad-puente también necesita de empresas de servicios específicos para el movimiento de bienes, como agencias aduanales, financieras de corto plazo, especialistas en comercio internacional, bufetes jurídicos, etc. La ciudad-punto de acceso, por el contrario, necesitará de residencias, eq:uipamientos educativos, instituciones culturales, áreas comerciales y de recreación, adecuadas para una fuerza de trabajo con un alto nivel de preparación y bien remunerada, formada por ejecutivos y profesionistas; requerirá también de una infraestructura sofisticada de comunicación con acceso a cualquier parte del mundo. 
Cada una de estas estrategias, y otras adicionales que no incluimos en este esquema, pueden ser analizadas en forma similar. Cuando una ciudad especifica una o más estrategias para su plan a futuro, debe comprometerse al cambio institucional y a realizar las inversiones requeridas para la realización de este futuro deseado. Examinaremos a continuación la experiencia de planificación estratégica de las ciudades de Canadá, Estados Unidos y Europa Occidental, ya que son éstas, las ciudades que hemos investigado a la fecha. Este repaso no es más que una sugerencia para el lector a partir de nuestros planteamientos, cómo se puede enfocar la clasificación y luego el análisis de ciudades particulares.

En América del Norte, tanto Buffalo como Detroit son ejemplos de ciudades-puentes, en el sentido de que cada una trata de desarrollar su ventaja como lazo entre las economías industriales de Ontario y las regiones de Estados Unidos. Ambas ciudades compiten directamente en el manejo del tráfico hacia el Sur de América. Por otra parte, Montréal, Toronto y Vancouver tratan de alcanzar una posición de ciudades-puerta de acceso, con relación a empresas de Europa, Asia y, ahora, México, que serían la base para la penetración en el mercado combinado de los Estados Unidos y Canadá.

Lyon es un ejemplo de ciudad europea que trata de colocarse como ciudad-puente entre el "Norte" y el "Sur", usando la ruta de transporte tradicional del río Ródano, así como la ventaja histórica de la localización de esta ciudad con relación a esta ruta. Otra ciudad que se perfila como una ciudad-puente es Sevilla, que espera así ligar la Unión Europea con Africa del Norte. La decisión reciente de construir un túnel en Tarifa, entre España y Marruecos, puede dar cierto viso de realidad a este sueño. Amsterdam y Copenhague han tratado activamente en las últimas décadas de ubicarse como ciudades-puerta de acceso, para competir con sus contrapartes más grandes y más desarrolladas, como Londres, París y Francfort. La figura 1, puede ser usada para explicar algunas de las decisiones específicas y las inversiones que cada ciudad debe hacer para ser exitosa en el desarrollo de su potencial estratégico.

Barcelona y Turín, así como Chicago, Cleveland y Montréal, son ciudades que han tomado la decisión de fundar su futuro, en buena medida, en sus bases manufactureras tradicionales. El acceso a los mercados externos es esencial para dichas ciudades, así como medidas, todas importantes, tales como las relaciones internacionales, la asistencia a las empresas más pequeñas y nuevas en sus esfuerzos por proyectarse internacionalmente, también los servicios empresariales especializados y las alianzas entre ciudades.

En este momento del desarrollo del ensayo, quizás sería más útil para el lector intentar aplicar este enfoque a las ciudades con las cuales está familiarizado, más que dar nuevos ejemplos sobre las ciudades que hemos estudiado. Pero debemos hacer todavía una observación más, con respecto a la planificación estratégica de las ciudades. Hasta el momento hemos desarrollado poco o nada en relación a la posible cooperación entre las ciudades; el aspecto competitivo de las relaciones entre las ciudades indudablemente ha dominado la discusión. Las ciudades que se encuentran en competencia directa entre sí, como por ejemplo Toronto y Montréal, (tratando de ser un punto de acceso para empresas europeas en América del Norte) o Bremerhaven y Rotterdam (que compiten en su posición de ciudades portuarias) no estarán muy dispuestas a compartir información o a emprender estudios conjuntos de sus problemas comunes. Sin embargo, no parecería ser el caso para las ciudades en continentes separados, y es justamente allí donde se pierde un vasto potencial para una cooperación fructífera.

En la discusión anterior sobre las ciudades y las estrategias, resultó claro que algunas ciudades europeas, canadienses y americanas están intentando, en continentes distintos, tener éxito con la misma estrategia; señalemos también que tienen poco que temer de las otras, desde el punto de vista de la competencia directa. En la figura 2, algunas de las posibilidades de cooperación intercontinental se hacen más explícitas. Los “retos estratégicos" enlistados abajo del cuadro, son una combinación de estrategias de la figura 1 y de los temas de mayor problema para las ciudades en relación con su internacionalización, como por ejemplo el estatuto periférico o la gobernabilidad efectiva. Lo que sugiere esta figura, es que mientras que Toronto y Montréal encontrarán imposible discutir entre sí la solución a sus problemas como ciudades-puntos de acceso, cada una por separado puede ser capaz de discutir esta temática con ciudades europeas como Copenhague o Amsterdam. Lo mismo se puede decir para las ciudadespuentes de Detroit y Buffalo y sus contrapartes europeas como Lyon, Hamburgo o Copenhague. Iniciativas como Las Ciudades de Invierno o el Congreso de Ciudades Portuarias son ejemplos de cooperación funcional entre ciudades, pero mucho más puede ser logrado si se sigue la trama conceptual de las figuras 1 y 2 .

Nuestra investigación no se ha extendido a las ciudades mexicanas, a pesar de que es el paso próximo más obvio. Algunos investigadores han realizado trabajos sobre las relaciones de cooperación transfronterizas, como las que surgen entre San Diego y Tijuana o entre El Paso y Ciudad Juárez. También algunos otros 
estudios han empezado a examinar las ciudades mexicanas en este contexto.?

Esperamos haber dejado clara nuestra concepción con respecto a que las ciudades individualmente pueden examinar sus posiciones estratégicas y pueden formular planes para su desarrollo económico y para la internacionalización, que les permitan alcanzar las metas deseadas por sus ciudadanos. El movimiento Eurocities ha logrado captar este sentir al iniciar el "Manifiesto de las Eurociudades” con la siguiente frase:

"Europa enfrenta actualmente un rápido proceso de integración política, económica y cultural. Mientras que los Estados han sido los protagonistas más importantes de la construcción europea, las regiones ban recibido progresivamente de la organización comunitaria, la atención que merecían... abora viene el turno de las ciu. dades" (Eurocities, 1989:9). ${ }^{8}$

\section{La competitividad}

Una aproximación menos centrada en los objetivos específicos de la formulación de políticas por parte de las economías urbanas, es el tema más general de la competitividad de las ciudades para enfrentar a otras ciudades en competencia. Mientras que la competitividad ha sido considerada, por mucho tiempo, como un tema de interés apropiado para las empresas y la economía nacional, en la economía globalizada descrita antes, una buena parte de la responsabilidad de la política en este tema, recae en el nivel del gobierno de la ciudad y en las entidades del sector privado local. Michael Porter ha reconocido esta situación en su libro La ventaja competitiva de las naciones, cuando afirma que "el proceso de crear capacidades y las influencias relevantes

7 Norris Clemente (San Diego State University) ha trabajado sobre el primer par de ciudades y Samuel Schmidt sobre el segundo. Daniel Hiernaux está trabajando sobre un amplio conjunto de ciudades mexicanas. Para aportaciones de los dos últimos, véase Kresl, Karl y Gary Gappert (1995), capítulos 9 y 5 respectivamente. Para San Diego, Tijuana, referirse a Clement, Norris (1993), San Diego-Tijuana in transition: a regional analysis, San Diego State University, Institute for Regional. Studies of the Californias, San Diego.

${ }^{8}$ Eurocities (1989), Eurocities, documents and Subjects of Eurocities Conference, 21 y $22 d e$ abril de 1989, Comité Organizador de la Conferencia Eurocities, Barcelona. para el nivel de mejoramiento y de innovación, son eminentemente locales" (Porter, 1990: 127)

Afortunadamente, la literatura sobre la competitividad económica es extensa y las variables que se sugiere tomar en cuenta, no respetan las fronteras nacionales o una división del trabajo y de responsabilidades entre los diversos niveles del gobierno. En el estudio de la competitividad de las ciudades que desarrollamos, se delimitaron dos proyectos separados pero interrelacionados. El primero es una revisión de la literatura que ha sido usada para identificar las variables clave y para sustentar una justificación teórica del uso de cada variable. ${ }^{9}$ El segundo, un análisis de regresión emprendido con Balwant Singh, usa información de las cuarenta ciudades más grandes de los Estados Unidos. ${ }^{10}$ Elegimos a los Estados Unidos para este primer estudio empírico y comparativo, por la simple razón de la existencia de información comparable para un gran número de ciudades. ${ }^{11}$ La revisión en sí no implica discusión en la medida en que sus resultados se reflejan en el trabajo empírico.

El primer paso, en el estudio empírico de las cuarenta ciudades, fue el de establecer un rango de ciudades en función de su competitividad. Para ello, se usó información sobre el crecimiento a través de tres variables: el valor agregado generado por los servicios al productor, las ventas minoristas y la manufactura, todo durante la década 1977-1987, ésta fue la información más reciente que se podía conseguir en el momento de realizar el estudio. El segundo paso fue la determinación estadística de las variables que explicarían mejor la clasificación de las ciudades. La teoría económica señala que la competitividad urbana es función de dos conjuntos de variables determinantes: las económicas y las estratégicas. Las variables estratégicas incluidas fueron: la eficiencia del gobierno, la estrategia urbana seleccionada, la cooperación entre sectores privados y públicos, y la flexibilidad institucional. Las respuestas de las oficinas de alcaldes que obtuvimos fueron de la mitad de las cuarenta ciudades, de tal suerte

' V'éase Kresl, Peter, "The determinants of Urban Competitiveness, a survey", en: Kresl, Karl y Gary Gappert (ed.) (1995: 45-68).

${ }^{10}$ Kresl, Peter Karl y Balwant Singh (1995), "The competitiveness of cities: The United States", en: OCDE (1995: 424-446).

"I El Prof. Singh y el autor de este trabajo, estamos trabajando en una actualización del estudio usando datos más recientes, $\mathrm{y}$ haciendo ajustes al modelo a la luz de investigaciones recientes. 
que será necesario un análisis posterior para poder incluir estos determinantes en el análisis.

Los determinantes económicos incluyen: los factores de la producción, lainfraestructura, lalocalización, laestructura económica y las facilidades urbanas. La información utilizada dependió en parte, de los años para los cuales estaba disponible. Como se puede notar, mientras que los determinantes estratégicos son cualitativos en su esencia y se obtienen a partir de trabajo de campo, los determinantes económicos son cuantitativos, y pueden ser obtenidos mediante los censos publicados regularmente o por reportes u otras fuentes secundarias. En nuestro análisis, hemos puesto a prueba diversos conjunto alternativos de variables que representan los determinantes económicos individuales y hemos podido demostrar que los que tienen mayor representatividad estadística son los siguientes:

- El incremento del ingreso per cápita entre 1980 y 1990

- El incremento en la participación de la población de más de 25 años con nivel educativo de licenciatura, entre 1980 y 1990

- El número de centros de investigación dividido por la fuerza de trabajo en 1987 ,

- El porcentaje de la fuerza de trabajo en "ejecutivos y profesionistas" en 1990

- Una variable binaria (dicotómica) para la localización en el "cinturón del sol" (Sunbelt) y el Oeste, en oposición con las ciudades de base industrial ubicadas en el Norte,

- La porción de la fuerza de trabajo en "ingeniería, contabilidad, investigación y gestión” (en resumen "ingeniería e investigación") en 1990, y

- El rango de la ciudad en instituciones culturales en 1985.

Cada una de estas variables resultó ser significativa con un grado de confiabilidad de 0.05 , con excepción de la primera que alcanzó una confiabilidad de 0.10; el R cuadrado para la ecuación resultante en su totalidad fue de $73.5 \%$
La mayor parte de las variables pueden ser consideradas independientes en su naturaleza, por ejemplo la localización en el "cinturón del sol" o en el "Norte industrial". Sin embargo, dos de estas variables exigieron mayor explicación, por lo que hicimos un análisis complementario para asegurar su determinación:

'1. El crecimiento del ingreso per cápita, y

2. El componente "ingeniería e investigación" de la fuerza de trabajo.

Este análisis nos agregó cuatro variables más a la lista original:

- El incremento en la participación de las empresas con más de 100 trabajadores entre 1977 y 1987.

- El número de centros de investigación diferenciado por el valor agregado de la manufactura en 1987 ,

- Elporcentaje de crecimiento de lainversión en infraestructura y equipamiento entre 1977 y 1985 , y

- El número de instituciones culturales en 1985.

El resultado de este análisis fue un conjunto de once variable que son estadísticamente representativas para explicar la clasificación de las cuarenta ciudades sobre la base de su competitividad.

El hecho de identificar estos "determinantes de la competitividad urbana” es en sí, un resultado de interés. Sin embargo, también hemos demostrado que se puede usar la posición de cada ciudad con relación a su competitividad o en cada una de sus variables determinantes para dos propósitos:

1. Evaluar la estrategia de desarrollo económico puesto en práctica por la ciudad.

2. Diseñar una estrategia de desarrollo económico que incremente al máximo la competitividad de la ciudad.

Una ciudad ubicada en un rango aproximado de quince o de veinte en su competitividad, puede ser considerada como deficiente con relación a otras ciudades en competencia, por lo que se refiere a los equi- 
pamientos culturales, las facilidades para la investigación y las empresas de 100 empleados o más, por ejemplo. Un caso que arroja nuestro estudio es el de la ciudad de Phoenix, en Arizona. Phoenix se ubica en la posición 10 en competitividad, pero en la 20 y la 24 dentro de las variables de centros de investigación, en la 31 y la 33 en las dos variables relacionadas con las instituciones culturales, en la $18.5 \mathrm{en} \mathrm{el}$ porcentaje de la fuerza de trabajo con un grado universitario y finalmente, en la posición 24 en lo referente al crecimiento de las empresas con 100 trabajadores o más. Cada una de estas posiciones bajas (la primera siendo la mejor y la cuarenta la peor) indica un nicho en el cual la ciudad es deficiente con relación a otras ciudades en ciertos aspectos, que se demostró eran significativos para la competitividad de una ciudad. Si Phoenix busca subsanar estas deficiencias específicas (las instituciones culturales y de investigación, la atracción a empresas más grandes y más fuerza de trabajo con nivel universitario) sería capaz de mejorar su posición de competitividad en varios puntos. De tal suerte, las ciudades pueden usar este enfoque para lograr criterios objetivos para la determinación de los elementos más efectivos, en un plan estratégico.

\section{Un ejemplo actual de la necesidad de pensar en la competitividad}

De la experiencia reciente de la ciudad de Nueva York, surge una nueva evidencia: la necesidad de que las ciudades atiendan y destinen recursos para la planificación de su futuro, en un contexto económico globalizado. Entre 1993 y 1994, la autoridad portuaria de la región Nueva York-Nueva Jersey sacó a la luz dos reportes sobre "el turismo y las artes en la región Nueva York-Nueva Jersey" (Port Authority.., 1993 y 1994). El estudio reconoció que la economía metropolitana de Nueva York ha pasado por un decrecimiento de su sector manufacturero por varias décadas, así como por un crecimiento del sector de servicios. También reconoció que en Nueva York, los servicios financieros y la producción están entre los más importantes del mundo, y que son estas actividades las que han sido de gran relevancia para la situación del empleo y el ingreso en la ciudad. Un aspecto al cual se ha dado menos reconocimiento, es la creciente relevancia de las actividades relacionadas con el turismo y las artes en esta ciudad, que ahora representan 375,000 empleos y un impacto económico sobre la región metropolitana de 30 mil millones de dólares. Ambos estudios ponen de manifiesto la necesidad de poner en práctica políticas que garanticen la competitividad, en el contexto de los retos que significan otras áreas urbanas, de tal suerte que el informe sobre las artes concluye con lo siguiente: "las artes y las personas que las crean, las exhiben y las venden, significan una ventaja competitiva central para Nueva York y sus suburbios frente a nuestros competidores nacionales $e$ internacionales, en la lucha por la supervivencia en el próximo siglo" (Port Authority, 1993 y 1994: 10)

Con una preocupación evidente, dos años después, el Nere York Times discutió el contenido de los estudios encargados por la Comisión Presidencial de las Artes y las Humanidades y el Fondo Nacional de las Artes, bajo el título "Audiencias envejecidas apuntan a un Futuro oscuro para las Artes" (New York Times, 1996:A1, 4). Los cambios demográficos, las transformaciones en las preferencias sobre recreación y ocio de los grupos más jóvenes, así como las respuestas inadecuadas de las instituciones artísticas configuran unas nubes sombrías sobre el futuro del sector de las artes en Nueva York y otras ciudades de los Estados Unidos. En otros dos reportajes importantes, surgidos en la semana siguiente al ya mencionado, se discutieron las perspectivas de largo plazo para la región metropolitana de Nueva York, usando un informe de la Asociación del Plan Regionall como base del análisis (NYT, 1996: feb, 14: A1, 1 y feb. 19, A1, 6). La Asociación reporta que: "si las tendencias actuales se mantienen, los 31 condados de la región metropolitana de Nueva York podrían entrar en una declinación económica a largo plazo en el próximo siglo". Las recomendaciones de la Asociación fueron que: "la región debe reconstruir los centros urbanos, conectar los sistemas de transporte actual mente fragmentados para recrear un modelo integrado y reestructurar los sistemas de educación local" (NUT, feb. 14, 1996: A1, 6). Para lograr esto, sería necesario un nuevo modelo de gobierno regional.

Nueva York puede representar el ejemplo más difundido de una ciudad que se ve forzada, por las condiciones cambiantes de la competitividad, a estudiar su situación y a planificar su futuro; aunque es muy notorio que esta condición necesaria no es la única. Nuestro trabajo de campo en las alcaldías de cuarenta grandes ciudades de los Estados Unidos, revela que muchas ciudades se ven en una posición bastante similar. Sus economías tradicionales están afectadas por la liberalización del comercio, el cambio tecnológico y la globalización, en tanto que los líderes de las ciudades usualmente reconocen la necesidad de dar una respuesta activa a esta situación. Se ha constituido, inevitablemente, en una cuestión de diseño de una estrategia apropiada, así como también en la necesidad de alcanzar un sistema de 
gobierno que sea suficientemente eficaz para implementar la estrategia con todo su potencial.

\section{El futuro de las ciudades}

La primacía del Estado-nación se ve retada desde diversas perspectivas. Kenichi Ohmae plantea que los Estados-naciones, sencillamente, cada día se hacen menos relevantes, por lo que escribió: "En un mapa político, las fronteras entre los países son tan evidentes como siempre. Pero en un mapa de la competencia, aquel que muestre los flujos reales de la actividad financiera e industrial, estas fronteras están siendo fuertemente desdibujadas" (Ohmae, 1990:18). En un número de la revista Daedalus, dos autores presentan un panorama más matizado o complejo. Vincent Cable anota que "La historia nos ha demostrado que mucho de lo que consideramos como un avance inexorable de la globalización es de becho frágil y reversible. Sin reglas fuertes a nivel global (y regional) puede ser llevado hacia atrás" fácilmente; advierte también que si las reglas y las instituciones globales (como la Organización Mundial del Comercio y el FMI) demuestran ser ineficientes o insuficientes “...podríamos asistir a la resurrección en vez de a la muerte del Estadonación" (Cable, 1995 : 22-53). Por su parte, Vivien A. Schmidt argumenta que la competencia se construye entre las empresas productivas y el gobierno, más que entre los niveles nacionales y supranacionales de gobierno. La globalización está forzando a una reestructuración compleja del valor de todas las instituciones hasta en sus último componentes como son los individuos, que a la vez son entes económicos y políticos. La nota de advertencia de Schmidt destaca que "salvo que los Estados-naciones logren hacer sentir a sus ciudadanos que están participando en las decisiones supranacionales que afectan crecien temente sus vidas, la legitimidad tanto de las organizaciones supranacionales y sus Estados-naciones, se verá cuestionada en forma creciente” (Schmidt, 1995: pp. 75-106)

Las funciones, los grados de libertad y las atribuciones legales de las ciudades se van a ver afectadas por la forma según la cual la globalización altera las responsabilidades relativas y absolutas de las naciones, las empresas y las entidades supranacionales. Por ejemplo en Europa, Bruselas ha emergido como una fuente importante de financiamiento para las iniciativas locales y las ciudades pueden, en grados diversos, tratar directamente con la Unión Europea sin pasar primero por sus gobiernos nacionales. El Consejo de las Regiones, creado por el Tratado de Maastricht, y el movimiento de las Euro Cities son indicadores institucionales de la relevancia que tienen la ambición y la capacidad de acción autónoma que manifiesta el pensamiento de los líderes de las entidades subnacionales. En América del Norte, este desarrollo no ha seguido el mismo curso, ni ha sido expresado explícitamente, debido a las diferencias en las estructuras de gobierno, a la falta de interés en intervenir más allá de un relativamente bajo nivel de integración (un área de Libre Comercio), a un sentido permanente de competencia y de acción individualista en las ciudades de los Estados Unidos, y finalmente, una falta de conexión entre las ciudades de Canadá, México y Estados Unidos. Sin embargo, existe una oportunidad considerable para la toma de participación activa de las ciudades. cuestiones:

En síntesis, en este trabajo, hemos intentado demostrar tres

1. Como consecuencia de la liberalización del comercio y los cambios en la tecnología, las ciudades han surgido como actores económicos de primera importancia, hecho que se da paralelamente a una disminución marginal del papel de los Estados-naciones.

2. Las ciudades tienen la capacidad de analizar su situación en la economía global y de diseñar una respuesta estratégica a los retos y a las oportunidades inherentes a esta situación.

3. Las ciudades pueden poner en práctica políticas y realizar inversiones que les permitan desarrollar su mejor potencial para su futuro económico.

Hemos dado muchos ejemplos de la contribución que puede lograr la planificación estratégica y el mejoramiento de la competitividad, para asegurar que lo anterior sea efectivamente, posible para cualquier ciudad. Mientras que las ciudades de América del Norte han sido consultadas regularmente mediante instituciones como La Federación de Municipios Canadienses y La Liga Nacional de Ciudades, ${ }^{12}$ en relación a problemas urbanos, como el transporte, la pobreza, la criminalidad y las drogas, poco se ha hecho para resolverlos y mejorar la capacidad municipal frente a los problemas, los retos y las oportunidades inherentes a laglobalización, la competitividad urbana, la proyección internacional de las empresas pequeñas y medianas, la

$12 \mathrm{NdT}$. Asociación estadounidense de ciudades. 
reticularización funcional de las ciudades y una amplia gama de otros temas.

Es ahora virtualmente imposible para cualquier ciudad ignorar que ella misma y sus constituyentes económicos actúan en un medio global. Sólo queda por esperar que cuando esta creciente visión llegue a un nivel suficiente de comprensión y se reconozca la pesada carga que la globalización, el cambio tecnológico y la liberación del comercio plantean para las ciudades, se llegue a difundir lo suficiente este conocimiento, de tal suerte que los gobiernos de las ciudades y los líderes del sector privado respondan más o menos de la forma que aquí se sugirió. Aquellos que planean estratégicamente para el mejoramiento de su competitividad, seguramente tendrán futuros más satisfactorios que aquellos que ignoran esta oportunidad.

Cuadro 1

Un modelo de Planificación Estratégica de la

Economía Urbana

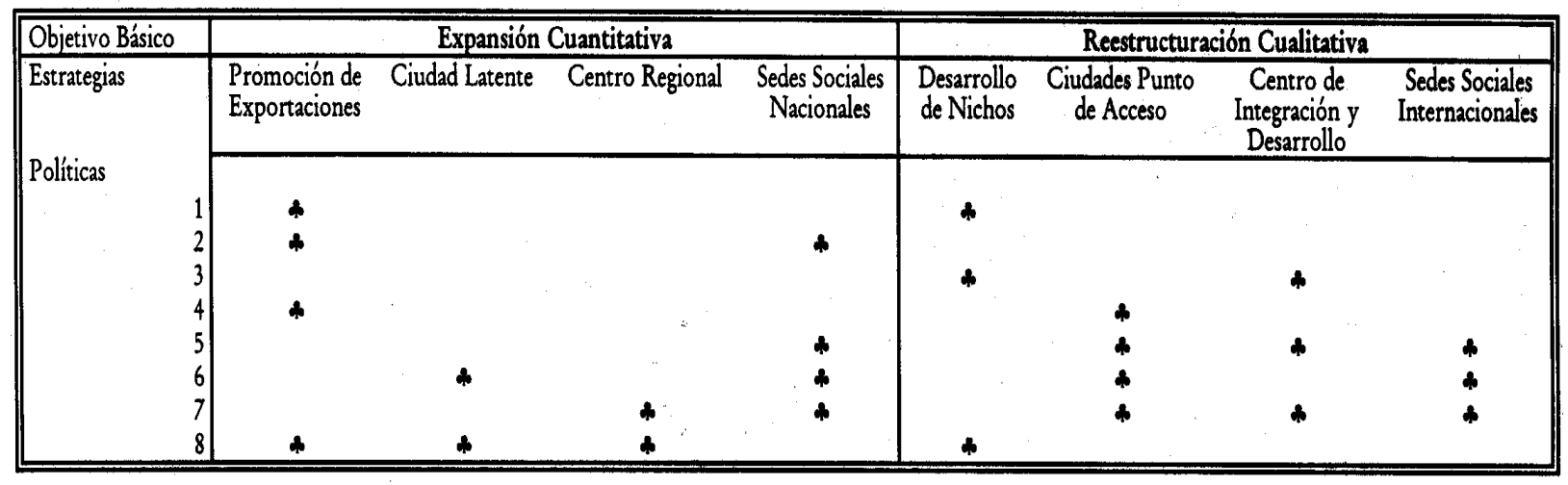

1 Desarrollo y apoyo de empresas pequeñas y medianas

2 Desarrollo de alianzas con otras ciudades

3 Establecimiento de lazos efectivos entre empresas, universidades y centros de investigación

4 Establecimiento y expansión de lazos internacionales

5 Construcción de infraestructura: Vivienda y Facilidades Urbanas

6 Construcción de infraestructura: Transporte

7 Construcción de infrestructura: Comunicación

8 Desarrollo de servicios adecuados, especializados para negocios

Fuente: Kresl, Peter Karl (1992), The Urban Economy and Regional Trade Liberalization, Nueva York; Praeger, p. 36. 


\section{Cuadro 2}

Posibilidades de cooperación entre ciudades

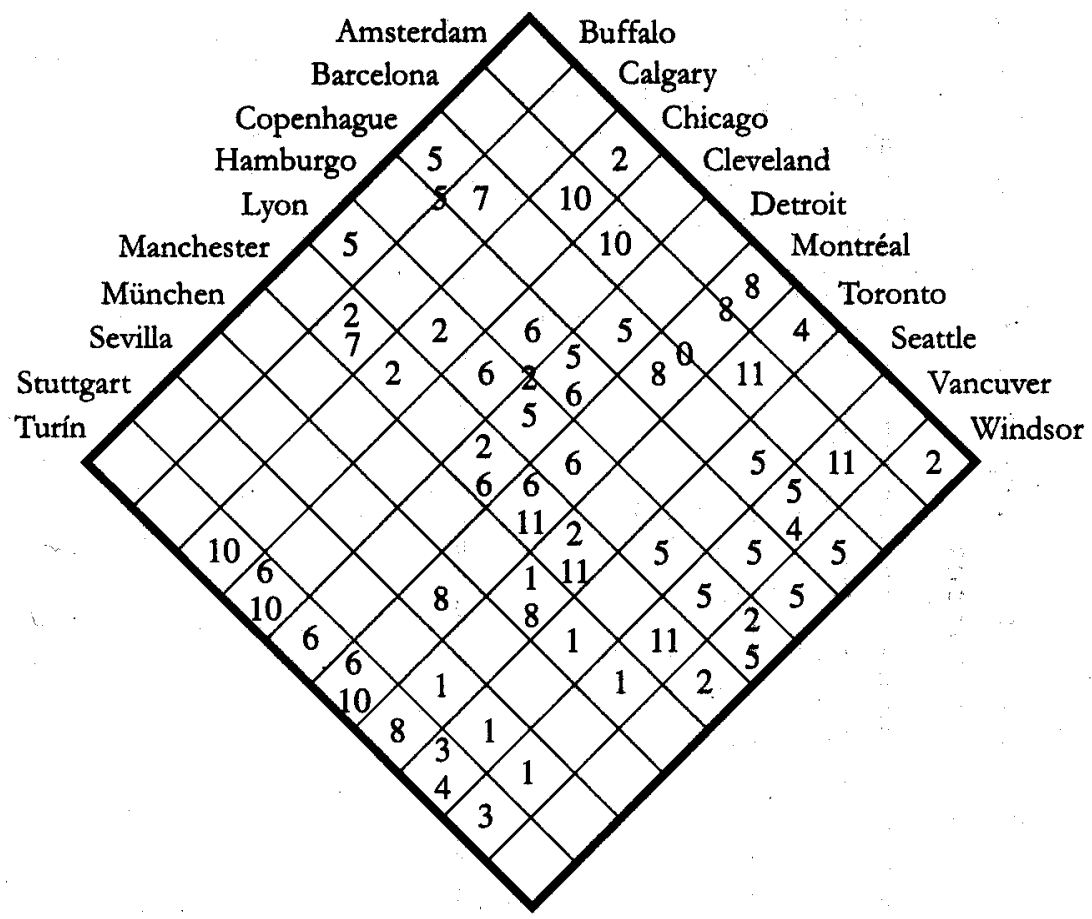

\section{Retos Estratégicos}

1. Gestión del crecimiento

2. Encontrar un nicho

3. Articulación de investigación y tecnología

4. Ciudades punto de acceso

5. Ciudades puente

6. Reticularización de ciudades

7. Enfrentar el estatuto de periferia

8. La ciudad como centro de la nación

9. Ciudad de sedes sociales

10. Vitalidad de la manufactura

11. Gobierno efectivo 


\section{Bibliografía}

Cable, Vincent (1995), "The Diminished Nation-State: A study in the Loss of Economic Power".

Clement, Norris (1995), San Diego-Tijuana in transition: A regional analysis, San Diego State University, Institute for Regional Studies of the Californias, San Diego.

Daedalus, (primavera, 1995), Volumen 124, Número 2, pp. 23-53.

Eurocities Movement (1989), Eurocities (Documents and Subjects of Eurocities Conference, 21 and 22 Abril, 1989), Comité Organizador del Coloquio "Eurocities", Barcelona.

Harrison, Bennett (1995), "Industrial Districts: Old Wine in New Bottles?", Regional Studies. Vol. 226, número 5, pp. 469-483.

Hiernaux, Daniel (1995), "Globalizing Economies and Cities: A view From Mexico", en: Kresl, Karl y Gary Gappert (eds.)., North American Cities and the Global Economy, Thousand Oaks: Sage Publications, pp. 112-129.

Kresl, Peter Karl and Balwant Singh (1995), "The Competitiveness of Cites: the United States", Cities and the New Global Economy, The Government of Australia and the Organization for Economic Cooperation and Development, Vol. 2, Melbourne.

(1995), "The Determinants of Urban competitiveness: A Survey", en: Peter Karl Kresl y Gary Gappert (eds.), North American Cities and the Global Economy, Thousand Oaks: Sage Publications, Urban Affairs Annual Review, No. 44.

Krugman, Paul (1991), Geography and Trade, MIT Press, Cambridge.

Ohmae, Kenichi (1990), The Borderless World, Harper Collins, Nueva York. 
Organization for Economic Cooperation and Development (1995), Cities and the New Global Economy, Organization for Economic Cooperation and Development and the Commonwealth of Australia, Canberra.

The Port Authority of NY \& NJ. (1993), Tourism and The Arts in the New York-New Jersey Region: Part 1- The Arts as an Industry: Their Economic Importance to the New York-New Jersey Metropolitan Región, The Port Authority of NY \& NJ, New York.

- (1994), Tourism and The Arts in the New York:New Jersey Region: Part 2- Destination New York-New Jersey: Tourism and Travel to the Metropolitan Region, The Port Authority of NY $\&$ NJ, New York.

Porter, Michael (1990), The Competitive Advantage of Nations, The Free Press, New York.

Sassen, Saskia (1991), The Global City: New York, London, Tokyo, Princeton University Press, Princeton.

Schmidt, Samuel (1995), "Planning a U.S-Mexican Bi-National Metropolis: El Paso Texas-Ciudad Juárez, Chihuahua", en: Kresl, Karl y Gary Gappert (eds.), North American Cities and the Global Economy, Thousand Oaks: Sage Publications, pp. 187-220.

Schmidt, Vivian A (1995), "The New World Order, Incorporated: The Rise of Business and the Decline of the Nation-State", Daedalus, Primavera, Vol. 124, Número 2, pp. 75-106.

Varios autores (1991-1992), Ekistics: Urban networking in Europe, Vol. 58, Número 350/351, septiembre/octubre-noviembre/diciembre y Vol. 59 Número 352/353, enero/febrero-marzo/abril. 